\title{
Cerebral amyloid angiopathy-related inflammation: a case report presenting with a rare variant in SORL1 gene
}

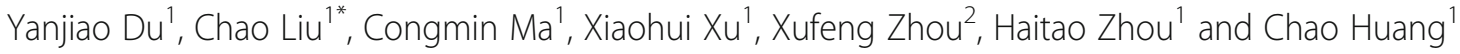

\begin{abstract}
Background: Cerebral amyloid angiopathy-related inflammation (CAA-ri) is a rare clinical entity, characterized by headaches, seizures, rapidly progressive cognitive decline, behavioral changes and magnetic resonance imaging (MRI) findings underlying the autoimmune and inflammatory reaction at the level of CAA-affected vessel. CAA-ri is likely responsive to corticosteroid. MRI shows asymmetric and multifocal white matter hyperintensity (WMH) lesions and multiple cerebral microbleeds. Apolipoprotein E (ApoE) $\varepsilon 4$ homozygosity is associated with CAA-ri strongly [Neurology 68(17):1411-1416, 2007, Ann Neurol 73(4):449-458, 2013, J Alzheimers Dis 44(4):1069-1074, 2015]. SORL1 processes a causal involvement in Alzheimer's disease (AD) as a proposed modulator of the amyloid precursor protein (APP). It is unclear whether SORL1 is involved with CAA-ri or not.

Case presentation: A 48-year-old woman suffered from a one-day history of a headache, nausea, and vomiting. Neurological examination revealed normal. We diagnosed this case as probable CAA-ri according to the clinic manifestations and MRI. Gene detection indicated a rare variant in SORL1 and ApoE $\varepsilon 4$ homozygosity. When treated with corticosteroid, the patient's clinical symptoms and MRI manifestations were almost relieved. However, when keeping the corticosteroid withdrawal for three months, the patient relapsed with a headache and typical images on MRI emerged. Corticosteroid therapy was effective again. Unfortunately, susceptibility weighted imaging (SWI) showed increased microbleeds. With tapering corticosteroid slowly, no recurrence was found on this patient with four-month follow-up.
\end{abstract}

Conclusion: A variant of SORL1 may be associated with CAA-ri, recurrence of disease could be detected with MRI by an increased microbleeds. Our case report suggests that corticosteroid therapy might be effective for CAA-ri.

Keywords: CAA-ri, ApoE, SORL1, Corticosteroid, Recurrence

\section{Background}

Cerebral amyloid angiopathy (CAA) is presented with progressive deposition of amyloid proteins within the cortical and leptomeningeal arteries, which is a common pathology in the elder [1, 2]. In recent years, studies show that coexisting inflammations found in CAA patients, such as vasculitis or perivasculitis, have been recognized as CAA-related inflammation (CAA-ri) [3]. CAA-ri is a rare clinical entity, characterized by headaches, seizures, rapidly progressive cognitive decline,

\footnotetext{
* Correspondence: liuchao-20001103@163.com

'Department of Neurology, Luoyang Central Hospital Affiliated to

Zhengzhou University, NO. 288, Middle Zhongzhou Road, Xigong Square,

Luoyang 471000, China

Full list of author information is available at the end of the article
}

behavioral changes and MRI findings underlying the autoimmune and inflammatory reaction at the level of CAA-affected vessel [4-7]. CAA-ri is thought to result from an inflammatory response to $\beta$-amyloid $(\mathrm{A} \beta)$ protein in the blood vessel walls [5] and likely responsive to corticosteroid or other immunosuppressive agents [7]. According to the pathology, CAA-ri can be divided into two subtypes. Inflammatory CAA is perivascular infiltration around the vessel wall, and A $\beta$-related angiitis is characterized by transmural and intramural inflammation, often with gulomas formation $[8,9]$. Amyloid-related imaging abnormalities (ARIA) represent the major severe side effect of $A \beta$ immunotherapy for Alzheimer's disease (AD), and characterize the acute phase of CAA-ri [9]. Hence, this supports a

(c) The Author(s). 2019 Open Access This article is distributed under the terms of the Creative Commons Attribution 4.0 International License (http://creativecommons.org/licenses/by/4.0/), which permits unrestricted use, distribution, and 
hypothesis that an immunological response to vascular amyloid, with increased vascular permeability, may be a common underlying pathophysiological mechanism of CAA-ri [10]. Apolipoprotein E (ApoE) $\varepsilon 4$ homozygosity known to associate with increased burden of $A \beta$ deposition in vessels [11], is strongly associated with CAA-ri $[4,5,12]$. In this case, we present a case of spontaneous CAA-ri with a variant in SORL1, which emerges as a promising candidate in control of the brain amyloidogenic processes in $\mathrm{AD}$ pathology [13] and with ApoE \&4. homozygosity.

\section{Case presentation}

A 48-year-old woman presented to our outpatient neurology with a headache, nausea, and vomiting for one day. Her headache was persistent, tolerable and blunt in the right temporal region. She had a hypertension history for three years and had been controlled within normal range by antihypertensive drugs. She had no psychosocial and familial hereditary history. Neurological examination was normal. Routine blood tests, serum C-reactive protein level and erythrocyte sedimentation rate showed normally. Serum thyroid hormone and sex hormone level were also in the normal range.
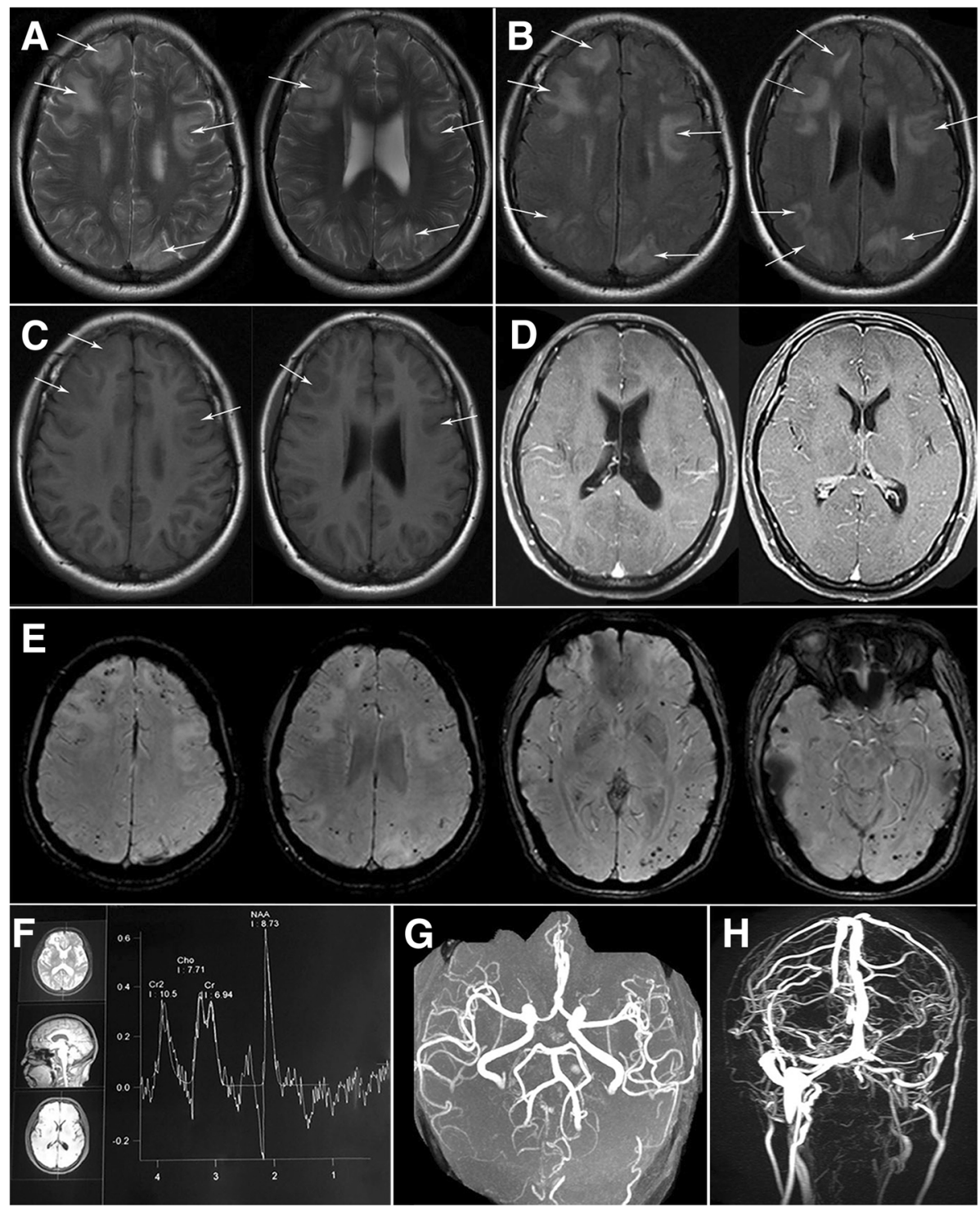

Fig. 1 Brain magnetic resonance imaging (MRI) findings of our patient at the onset, a-c multifocal white matter hyperintensity lesions were revealed on T2 and fluid attenuated inversion recovery (FLAIR) and slight hypointense on T1 (arrows); $\mathbf{d}$ gadopentetate enhanced MRI showing no lesion was enhanced; e: susceptibility weighted imaging (SWI) showing multiple cerebral microbleeds in cortical and subcortical areas; $\mathbf{f}$ magnetic resonance spectrum demonstrating a normal spectrum in lesions; $\mathbf{g}$ magnetic resonance angiography was normal; $\mathbf{h}$ : magnetic resonance venography showing thinness in the bilateral transverse and left sigmoid sinus and decreased signal intensity 


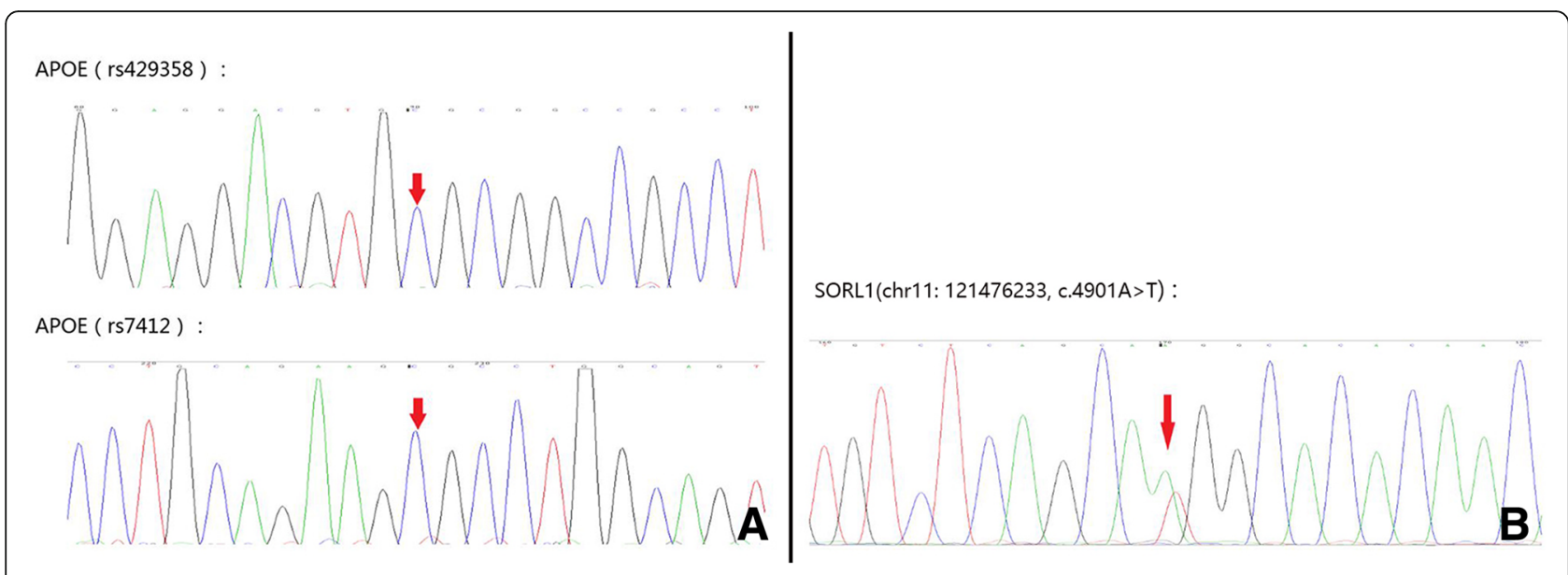

Fig. 2 Gene detection results of the patient. a Apolipoprotein E $\varepsilon 4$ homozygosity, b a variant in SORL1:C.4901A>T

Serum anti-MPO and P-ANCA were weakly positive, while other indicators including antinuclear, anti-SSA, anti-SSB, anti-dsDNA, anti-Sm, anti-RNP, anti-Scl-70, anti-RP3, anti-GBM, anti-neutrophil cytoplasmic antibodies in cytoplasmic, antibodies to neuro-paraneoplastic syndromes, neuromyelitis optica (NMO), myelin basic protein (MBP) and myelin oligodendrocyte glycoprotein (MOG) in blood as well as cerebrospinal fluid (CSF) were negative. Serum tumor markers were negative. Lumber puncture showed higher intracranial pressure with $240 \mathrm{mmH}_{2} \mathrm{O}$. Subsequent CSF analysis showed normal protein, glucose, and cell count. Testing on the bacterial, viral, fungal and cryptococcal pathogens of CSF was negative. Magnetic resonance imaging (MRI) of the brain revealed multifocal white matter hyperintensity (WMH) lesions (cortico-subcortical) on T2 and fluid attenuated inversion recovery (FLAIR) associated mass effect, which were slight hypointense on T1 and didn't enhance on gadopentetate enhanced MRI (Fig. 1a-d). Susceptibility weighted imaging (SWI) revealed multiple cerebral microbleeds in cortical and subcortical areas (Fig. 1e). Magnetic resonance spectrum (MRS) demonstrated a normal spectrum. (Fig. 1f). Magnetic resonance angiography showed normally (Fig. 1g). Magnetic resonance venogram showed thinness in the bilateral transverse and left sigmoid sinus and decreased signal intensity (Fig. 1h). Further digital subtraction angiography excluded venous sinus thrombosis. The genetic sequencing showed ApoE $\varepsilon 4$ homozygosity and a variant in SORL1 (Fig. 2a-b).

Based on these findings, we made a diagnosis of probable CAA-ri. We firstly treated the patient with dexamethasone $10 \mathrm{mg} /$ day via venous for three days and then reduced it by half for three days, followed by oral prednisolone $60 \mathrm{mg} /$ day tapered within six weeks (a weekly reduction of $10 \mathrm{mg}$ ). On the 3rd day of treatment, her headache was significantly relieved. MRI demonstrated that the lesions on FLAIR almost completely disappeared

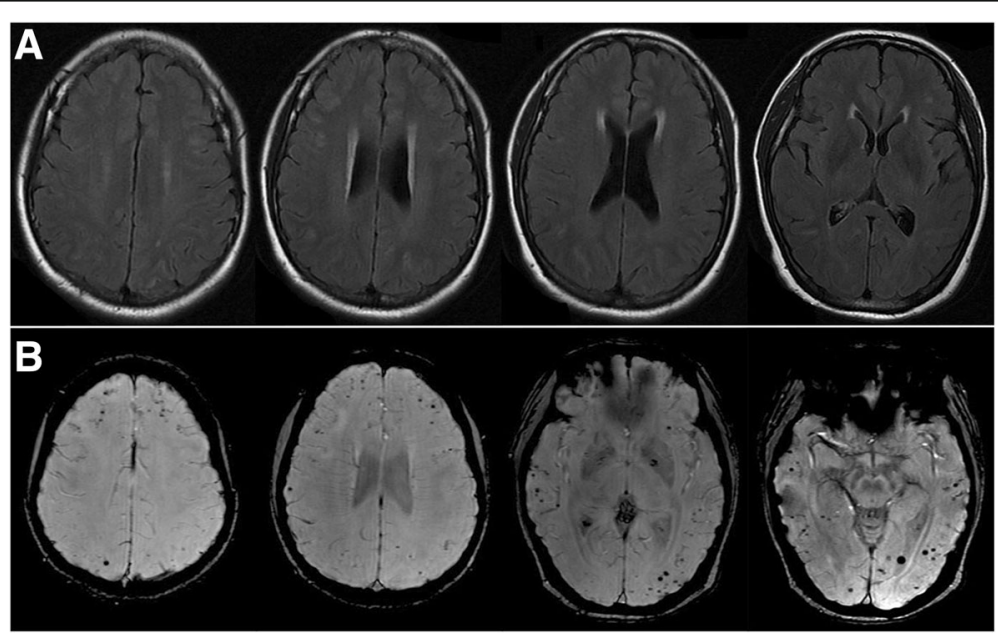

Fig. $3 \mathrm{MRI}$ findings after the first corticosteroid therapy. a FLAIR images showing lesions were almost completely disappeared; b SWI images showing multiple cerebral microbleeds remained unchanged 


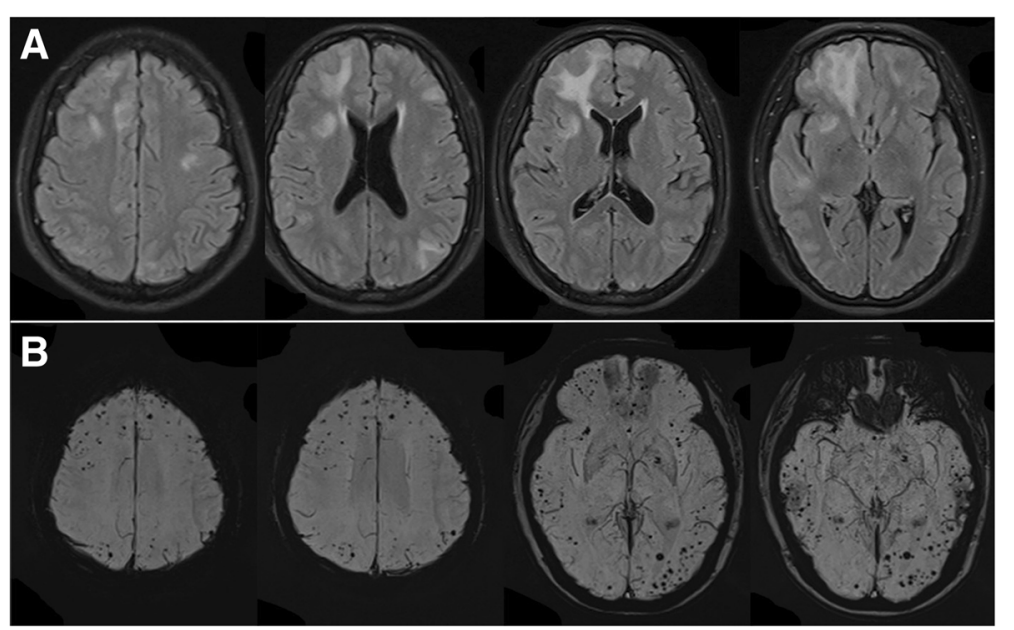

Fig. 4 Recurrence MRI images after corticosteroid withdrawal for three months. a multifocal white matter hyperintensity lesions were revealed on FLAIR; $\mathbf{b}$ multiple cerebral microbleeds in cortical and subcortical areas increased on SWI

six weeks later (Fig. 3a-b). She presented to our clinic with complaints of a headache again after corticosteroid withdrawal for three months. MRI indicated CAA-ri relapsed and SWI showed that the microbleeds in cortical/subcortical areas were increased (Fig. 4a-b). We launched the methylprednisolone $1000 \mathrm{mg}$ pulse therapy for her. Lesions on T2/FLAIR disappeared quickly. We recommended reducing the dose of corticosteroid gradually until a long-term administration of oral prednisone $5 \mathrm{mg} /$ day. No recurrence was found and the lesions of microbleeds were not increased any more over the next four-month follow-up (Fig. 5a-b).

\section{Discussion and conclusions}

Definitive diagnosis of CAA-ri generally requires typical clinical manifestation, typical MRI features, and brain biopsy [8]. A study demonstrated image-guided stereotactic brain biopsy is usually safe, but complications may occur, with transient and permanent morbidity in 9 and $13 \%$ of cases [14]. Considering the risk of brain biopsy, Auriel, et al. validated the modified probable CAA-ri criteria, which is sufficiently specific to be incorporated into the clinical practice for identifying patients with good sensitivity and excellent specificity [15]. According to the criteria [15], we made a diagnosis of probable CAA-ri based on five aspects: (1) the patient's age was over 40 years old; (2) the patient presented with headache but no acute intracerebral hemorrhage (ICH) was found; (3) MRI showed WMH lesions (cortico-subcortical) which were asymmetric without past ICH; (4) SWI revealed multiple cerebral microbleeds in cortical and subcortical areas; (5) No neoplastic, infectious or other
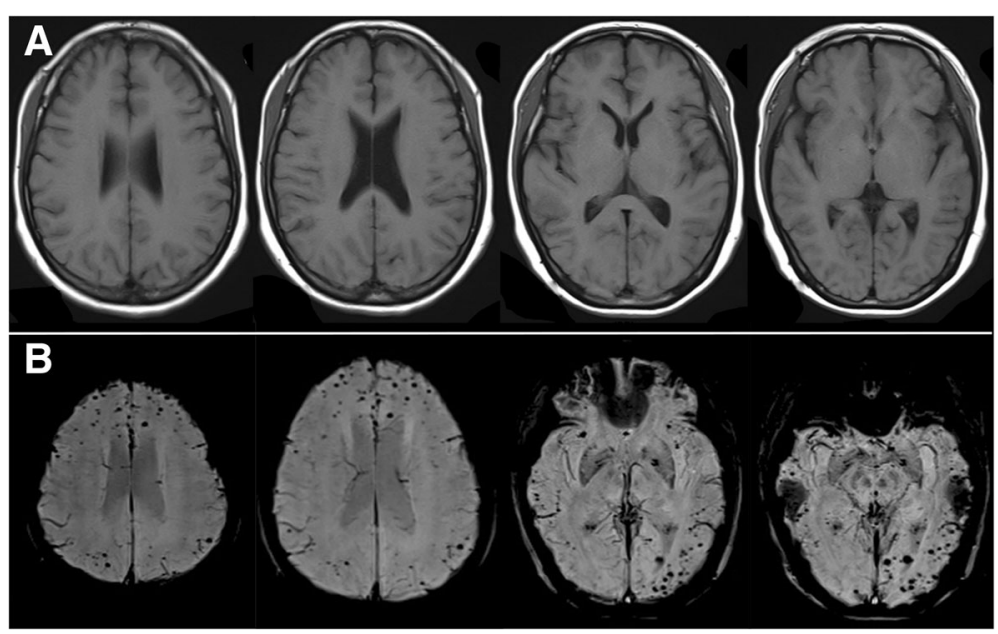

Fig. 5 MRI images after the second corticosteroid therapy for four months. a no lesion was found on FLAIR; $\mathbf{b}$ microbleeds were not increased on SWI 
indications were found according to the laboratory and imaging results. Lesions on T2/FLAIR revealed obvious edema and no lesion was enhanced in MRI gadolinium enhancement, which was similar to low-grade glioma and lymphoma [16]. However, they were excluded by a further MRS examination. The images of posterior reversible encephalopathy syndrome (PRES) was similar to CAA-ri. But PRES is always accompanied by hypertension combined with severe underlying diseases, including high blood pressure, pregnancy eclampsia, severe kidney disease, malignant tumor, systemic lupus erythematosus, etc. Due to the patient's controlled normal blood pressure and none of the diseases mentioned above, we excluded PRES. The diagnosis of CAA-ri is considered as definite if, in addition to these criteria, a histopathologic examination indicates perivascular, transmural, or intramural inflammation and amyloid deposition within vessels of the affected area in the cortex and the leptomeninges [7]. However, our patient refused to take a brain biopsy.

The best therapy of CAA-ri remains to be determined. The presented data support to use the empirical immunosuppressive therapy (and avoiding brain biopsy) for patients who meet the criteria proposed for probable CAA-ri $[7,15]$. A reasonable subsequent approach would be considered a brain biopsy in empirically treated patients who fail to respond to the corticosteroid therapy within three weeks [7]. Strict blood pressure control was also managed for patients with hypertension [17]. The primary treatment for our patient was dexamethasone $10 \mathrm{mg}$ per day via venous infusion. Clinical symptoms and image almost completely relieved (Fig. 3). After keeping corticosteroid withdrawal for three months, the patient developed recurrence. She was treated with high-dose methylprednisolone, clinical symptoms and lesions on T2/ FLAIR were completely relieved, which supported the diagnosis of CAA-ri $[4,6,7]$. When recurred, SWI showed increased microbleeds in the cortical/subcortical areas (Fig. 4). In the following four months, the lesions of microbleeds have not been increased, indicating that the microbleeds may be the result of the acute vascular inflammation of CAA-ri. To improve prognosis, large samples studies to further determine a better administration for CAA-ri are necessary.

Genetic detection of the patient indicated ApoE \&4 homozygosity and a variant in SORL1 (chromosome11, c.4901A>T) (Fig. 2), which was fairly rare in the normal population. Strong support for a causal involvement in neurodegenerative disease came with genetic studies associating SORL1 gene variants with the occurrence of sporadic AD [13]. SORL1 encodes a $250-\mathrm{kDa}$ protein named sorting protein-related receptor with A-type repeats (SorLA), decreasing production and deposition of $A \beta$ peptide as a proposed modulator of amyloid precursor protein (APP) processing $[13,18]$. A $\beta$ levels are mainly determined by the kinetic of SorLA and APP interaction according to the mathematic model which supports the fact that APP sorting pathway is a very important factor in defining the risk of $\operatorname{AD}[13,19,20]$. CAA-ri is thought to result from an inflammatory response to $A \beta$ protein in the blood vessel walls [5]. Spontaneous ARIA and CAA-ri have also been described in familial forms of $A D(F A D)$, i.e., in $\beta$-amyloid protein precursor -amyloid protein precursor $(\mathrm{A} \beta \mathrm{PP})$ duplication carriers [21], in presenilin 1-associated FAD (I202F PSEN1 mutation) [12], and in two siblings carrying the P284S PSEN1 mutation [22]. Thus, SORL1 may be associated with CAA-ri. A variant in SORL1 may give rise to the risk of CAA-ri via immune response to $A \beta$ excessive deposition. ApoE $\varepsilon 4$ homozygosity known to associate with increased burden of $A \beta$ deposition in vessels [11] is strongly associated with CAA-ri $[4,5,12]$ and may potentiate the intense inflammatory response [23]. Investigation of the degree of CAA-ri and inflammation in SORL1 mutation with ApoE $\varepsilon 4$ homozygosity would be an interesting avenue for future research.

A variant of SORL1 may be associated with CAA-ri, recurrence of disease could be detected with MRI by an increased microbleeds. Our case report suggests that corticosteroid therapy might be effective for CAA-ri.

\section{Abbreviations \\ AD: Alzheimer's disease; ApoE: apolipoprotein E; APP: amyloid precursor protein.; ARIA: Amyloid-related imaging abnormalities; $A \beta$ : $\beta$-amyloid; AßPP: $\beta$-amyloid protein precursor; CAA: cerebral amyloid angiopathy, CAA- ri: cerebral amyloid angiopathy-related inflammation; CSF: cerebrospinal fluid; FLAIR: fluid attenuated inversion recovery; ICH: intracerebral hemorrhage; MBP: myelin basic protein; MOG: myelin oligodendrocyte glycoprotein; MRI: magnetic resonance imaging; MRS: magnetic resonance spectrum; NMO: neuromyelitis optica; PRES: posterior reversible encephalopathy syndrome; SorLA: sorting protein-related receptor with A-type repeats; SWI: susceptibility weighted imaging; WMH: white matter hyperintensity}

\section{Acknowledgements}

We thank the patient for data collection and Kangso medical inspection for technical assistance. We thank Zhihui Duan, Yuezhen Han, Yan Li and Yonggang Wang for making some useful suggestions on polishing the manuscript.

\section{Funding}

None.

Availability of data and materials

All data and material supporting our findings are contained within the manuscript.

Authors' contributions

YD was a major contributor in writing the manuscript. $X Z, Y D, C L$ and $C M$ analyzed clinical and MRI manifestations. $\mathrm{HZ}$ and $\mathrm{CH}$ analyzed gene detection results. $\mathrm{YD}, \mathrm{CL}, \mathrm{CM}, \mathrm{XX}, \mathrm{HZ}$ and $\mathrm{CH}$ gave the clinical information containing medical history, neurological and MRI image findings, gene detection results and treatment. YD and CL contributed to editing and revising the manuscript and supervised this publication. All authors read and approved the final manuscript. 


\section{Ethics approval and consent to participate}

The Institutional Review Board of Luoyang Central Hospital Affiliated to Zhengzhou University approved this study. Informed consent for participation was obtained from the patient.

\section{Consent for publication}

Written informed consent was obtained from the patient for publication of this case report and any accompanying images. A copy of the consent is available for review by the editor of this journal.

\section{Competing interests}

The authors declare no conflicts of interest with respect to the research, authorship, funding, and/or publication of this article.

\section{Publisher's Note}

Springer Nature remains neutral with regard to jurisdictional claims in published maps and institutional affiliations.

\section{Author details}

'Department of Neurology, Luoyang Central Hospital Affiliated to Zhengzhou University, NO. 288, Middle Zhongzhou Road, Xigong Square, Luoyang 471000, China. ${ }^{2}$ Department of Radiology, Luoyang Central Hospital Affiliated to Zhengzhou University, NO. 288, Middle Zhongzhou Road, Xigong Square, Luoyang 471000, China.

Received: 29 September 2018 Accepted: 3 May 2019 Published online: 15 May 2019

\section{References}

1. Oh U, Gupta R, Krakauer JW, Khandji AG, Chin SS, Elkind MS. Reversible leukoencephalopathy associated with cerebral amyloid angiopathy. Neurology. 2004;62(3):494-7.

2. Charidimou A, Gang Q, Werring DJ. Sporadic cerebral amyloid angiopathy revisited: recent insights into pathophysiology and clinical spectrum. $J$ Neurol Neurosurg Psychiatry. 2012;83(2):124-37.

3. Sakaguchi H, Ueda A, Kosaka T, Yamashita S, Kimura E, Yamashita T, et al. Cerebral amyloid angiopathy-related inflammation presenting with steroidresponsive higher brain dysfunction: case report and review of the literature. J Neuroinflammation. 2011:8(1):116.

4. Kinnecom C, Lev MH, Wendell L, Smith EE, Rosand J, Frosch MP, et al. Course of cerebral amyloid angiopathy-related inflammation. Neurology. 2007;68(17):1411-6.

5. Piazza F, Greenberg SM, Savoiardo M, Gardinetti M, Chiapparini L, Raicher L, et al. Anti-amyloid $\beta$ autoantibodies in cerebral amyloid angiopathy-related inflammation: implications for amyloid-modifying therapies. Ann Neurol. 2013;73(4):449-58

6. Eng JA, Frosch MP, Choi K, Rebeck GW, aSM G. Clinical manifestations of cerebral amyloid angiopathy-related inflammation. Ann Neurol. 2004;55: 250-6.

7. Chung KK, Anderson NE, Hutchinson D, Synek B, Barber PA. Cerebral amyloid angiopathy related inflammation: three case reports and a review. Neurol Neurosurg Psychiatry. 2011;82(1):20-6.

8. Chu S, Xu F, Su Y, Chen H, Cheng X, Yu J. cerebral amyloid angiopathy (CAA)-related inflammation: comparison of inflammatory CAA and amyloidß-related angiitis. J Alzheimers Dis. 2016;51(2):525-32.

9. Salvarani C, Morris JM, Giannini C, Brown RD, Christianson T, Hunder GG. Imaging findings of cerebral amyloid Angiopathy, Aß-related Angiitis (ABRA), and cerebral amyloid Angiopathy-related inflammation. Medicine. 2016;95(20):e3613.

10. DiFrancesco JC, Longoni M, Piazza F. Anti-A $\beta$ autoantibodies in amyloid related imaging abnormalities (ARIA): candidate biomarker for immunotherapy in Alzheimer's disease and cerebral amyloid Angiopathy. Front Neurol. 2015;6.

11. Greenberg SM, Rebeck GW, Vonsattel JP, Gomez-Isla T, Hyman BT Apolipoprotein E epsilon 4 and cerebral hemorrhage associated with amyloid angiopathy. Ann Neurol. 1995;38(2):254-9.

12. Ryan NS, Lashley T, Revesz T, Dantu K, Fox NC, Morris HR. Spontaneous ARIA (amyloid-related imaging abnormalities) and cerebral amyloid Angiopathy related inflammation in Presenilin 1-associated familial Alzheimer's disease. J Alzheimers Dis. 2015;44(4):1069-74.
13. Andersen OM, Rudolph IM, Willnow TE. Risk factor SORL1: from genetic association to functional validation in Alzheimer's disease. Acta Neuropathol. 2016;132(5):653-65.

14. McGirt MJ, Woodworth GF, Coon AL, Frazier JM, Amundson E, Garonzik I, et al. Independent predictors of morbidity after image-guided stereotactic brain biopsy: a risk assessment of 270 cases. J Neurosurg. 2005;102(5):897-901.

15. Auriel E, Charidimou A, Gurol ME, Ni J, Van Etten ES, Martinez-Ramirez S, et al. Validation of clinicoradiological criteria for the diagnosis of cerebral amyloid angiopathy-related inflammation. JAMA Neurology. 2016;73(2):197-202.

16. Ronsin S, Deiana G, Geraldo AF, Durand-Dubief F, Thomas-Maisonneuve L, Formaglio $\mathrm{M}$, et al. Pseudotumoral presentation of cerebral amyloid angiopathy-related inflammation. Neurology. 2016;86(10):912-9.

17. Arima H, Tzourio C, Anderson C, Woodward M, Bousser MG, Macmahon S, et al. Effects of perindopril-based lowering of blood pressure on intracerebral hemorrhage related to amyloid angiopathy the PROGRESS trial. Stroke. 2010;41(2):394-6.

18. Blechingberg J, Poulsen ASA, Kjolby M, Monti G, Allen M, Ivarsen AK, et al. An alternative transcript of the Alzheimer's disease risk gene SORL1 encodes a truncated receptor. Neurobiol Aging. 2018;71:266.e211-66 e224.

19. Schmidt V, Baum K, Lao A, Rateitschak K, Schmitz Y, Teichmann A, et al. Quantitative modelling of amyloidogenic processing and its influence by SORLA in Alzheimer's disease. EMBO J. 2012:31(1):187-200.

20. Alcantara JH, Lao AR, Ruivivar LA. Stability analysis of the ODE model representation of amyloidogenic processing in Alzheimer's disease in the presence of SORLA. Mol BioSyst. 2016;12(5):1468-77.

21. Chamard L, Wallon D, Pijoff A, Berger E, Viennet G, Hannequin D, et al. Amyloid-related imaging abnormalities in AbetaPP duplication carriers. J Alzheimers Dis. 2013;37(4):789-93.

22. Floris G, Di SF, Cherchi MV, Costa G, Marrosu F, Marrosu MG. Multiple spontaneous cerebral microbleeds and leukoencephalopathy in PSEN1associated familial Alzheimer's disease: Mirror of cerebral amyloid Angiopathy. J Alzheimers Dis Jad. 2015;47(3):535-8.

23. Moussaddy A, Levy A, Strbian D, Sundararajan S, Berthelet F, Lanthier S. Inflammatory cerebral amyloid Angiopathy, amyloid-beta-related Angiitis, and primary Angiitis of the central nervous system: similarities and differences. Stroke. 2015;46(9):e210-3.

Ready to submit your research? Choose BMC and benefit from

- fast, convenient online submission

- thorough peer review by experienced researchers in your field

- rapid publication on acceptance

- support for research data, including large and complex data types

- gold Open Access which fosters wider collaboration and increased citations

- maximum visibility for your research: over $100 \mathrm{M}$ website views per year

At BMC, research is always in progress.

Learn more biomedcentral.com/submissions 